\title{
Generation mechanism for VLF chorus emissions observed at a low-latitude ground station
}

\author{
A. K. Singh and K. Rönnmark \\ Department of Theoretical Space Physics, Umeå University, Umeå, SE-90187, Sweden \\ Received: 30 June 2003 - Revised: 21 November 2003 - Accepted: 11 December 2003 - Published: 14 June 2004
}

\begin{abstract}
A detailed spectral analysis of VLF chorus emissions observed at the low-latitude ground station Gulmarg (geomag. lat., $24^{\circ} 26^{\prime} \mathrm{N}$, geomag. long., $147^{\circ} 9^{\prime} \mathrm{E}, L=1.28$ ) during the strong magnetic activity on 7-8 March 1986 have been carried out, which shows that each chorus element originates from the upper edge of the underlying hiss band. To explain various temporal and spectral features of these emissions, a possible generation mechanism has been presented based on the backward wave oscillator regime of the magnetospheric cyclotron maser. On the basis of this model, we have computed various chorus parameters as well as some magnetospheric parameters affecting the generation process. A comparison of the observed chorus characteristics with the proposed generation mechanism shows a good agreement.
\end{abstract}

Key words. Magnetospheric physics (energetic particles, trapped) - Space plasma physics (wave particle interactions; waves and instabilities)

\section{Introduction}

Generation of chorus emissions is one of the most puzzling problems of VLF waves in the Earth's magnetosphere. These emissions are the most intense of all naturally generated VLF plasma wave emissions. Chorus are characterised by a sequence of discrete elements appearing as intense tones, of short duration, usually rising and often overlapping, occurring regularly in association with disturbed magnetospheric conditions (Helliwell, 1965; Sazhin, 1982; Sazhin and Hayakawa, 1992). Although it is generally accepted that their generation is connected with the electron cyclotron resonance of whistler-mode waves and radiation belt electrons (Helliwell, 1967), the mechanisms responsible for the origin of chorus and the formation of spectrum of separate elements are still a subject of active experimental and

Correspondence to: A. K. Singh

(abhay_s@ rediffmail.com) theoretical research (Smith et al., 1996; Trakhtengerts, 1999; Singh et al., 2000; Lauben et al., 2002; Santolik and Gurnett, 2003).

Unlike mid- and high-latitude emissions, low-latitude VLF emissions have not been used much for exploring the inner magnetosphere. The main reason for this is that the propagation characteristics of VLF emissions in the lowlatitude ionosphere are not properly known, since satellite and ground-based results are scarce. Hence, the mechanism of their generation source and propagation are far from being well understood (Lalmani et al., 2000; Singh et al., 2000). Therefore, a better understanding of the generation mechanism for these VLF emissions observed at low-latitudes would be useful for analysing the properties of high energy trapped electrons. During the course of our analysis of VLF data recorded during January 1986 to July 1986 at the low latitude ground station Gulmarg (geomag. lat. $24^{\circ} 26^{\prime} \mathrm{N}$, geomag. long. $147^{\circ} 9^{\prime} \mathrm{E} ; L=1.28$ ), we found some excellent records of chorus emissions which we reproduce here with a discussion of their probable generation mechanism.

Helliwell (1967) developed a phenomenological model of the generation of discrete emissions. The mechanism is based on the cyclotron resonance of energetic electrons with whistler mode waves, which manifest themselves as backward wave oscillators. In this model the idea of second order cyclotron resonance was first formulated and it explained numerous types of discrete emissions with different frequency versus time characteristics. Further analytical and computational calculations (Nunn, 1974) confirmed the idea of the second order resonance and permitted a connection to be made through nonlinear currents, between the parameters of a triggered emission and those of the initial quasimonochromatic whistler wave. Omura et al. (1991) have given a comprehensive review of the nonlinear theory of triggered emissions. The spectral forms of most of the chorus emissions could be explained similarly (Nunn, 1986), but for naturally occurring emissions the cause of beam formation is not clear, since the initial quasi-monochromatic whistler signal is absent in the chorus. 


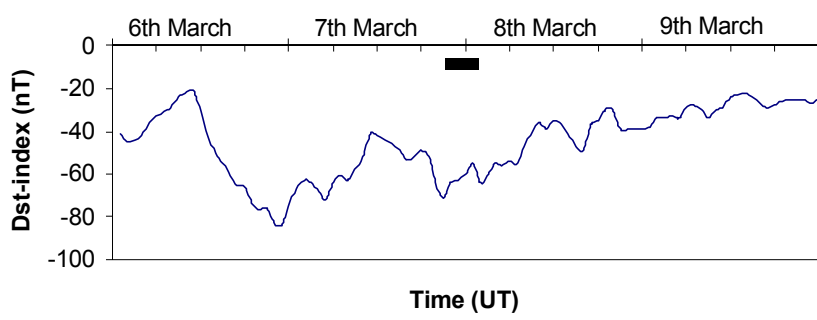

Fig. 1. The $D_{s t}$-index variations during the strong magnetic storm period of 6-9 March 1986. The duration of a chorus event is also marked.

Recently, Trakhtengerts $(1995,1999)$ suggested a generation mechanism of chorus emissions based on the backward wave oscillator (BWO) regime of magnetospheric cyclotron maser. He noticed that the presence of strong VLF hiss will cause pitch-angle scattering and precipitation of resonant electrons. This leads to the formation of a specific step-like deformation of the distribution function, which drastically changes all further developments of the wave-particle interactions (Trakhtengerts et al., 1996). Trakhtengerts (1995) revealed that the step-like deformation could be the cause of new generation regimes of the cyclotron instability, leading to a succession of discrete chorus signals.

In the present paper, first, we present a detailed spectral analysis of the VLF chorus emission events observed at Gulmarg during the routine recording of whistlers. We also review the generation mechanism for chorus emissions based on the backward wave oscillator regime of magnetospheric cyclotron maser (Trakhtengerts, 1995, 1999). An attempt is made to determine various chorus emission parameters as well as magnetospheric parameters, relevant to the generation process of chorus emissions. Finally, comparisons of the theory with observations are made.

\section{Chorus observations}

At the low-latitude ground station Gulmarg, the wideband VLF waves were received by a T-type antenna, suitably amplified by pre- and main-amplifiers and recorded using a tape recorder. The recorded VLF data were analysed by Sonograms and Advance VLF Data Analysis System (AVDAS). At low latitude, nights with observable VLF emissions are rather rare, and the activity is closely related to strong magnetic storms. However, during nights with chorus emissions the occurrence rate is comparable to that at mid latitude (Singh, 1993; Singh et al., 1999, 2000). Several interesting VLF events were recorded during the period January to July 1986 at the low-latitude ground station Gulmarg (geomag. lat. $24^{\circ} 26^{\prime} \mathrm{N}$, geomag. long. $147^{\circ} 9^{\prime} \mathrm{E}$ ). It was noticed that the VLF emissions recorded during a magnetic storm often are large enough to allow for a statistical analysis. The observations of chorus emissions at the low-latitude ground station Gulmarg are unusual in the sense that most of the reported

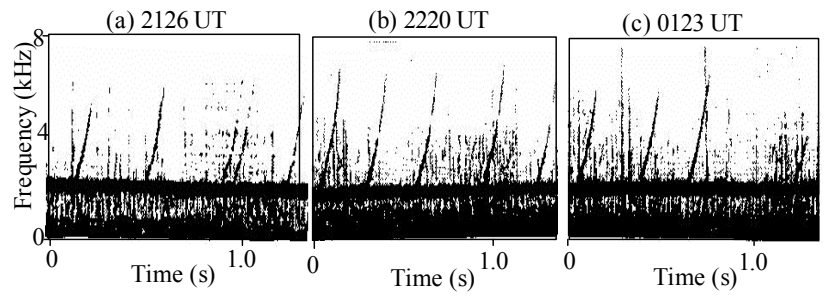

Fig. 2. The typical frequency-time spectrograms of chorus emissions observed at a ground station Gulmarg on 7-8 March 1986.

chorus observations are from satellites (Cornilleau-Wehrlin et al., 1978; Hattori et al., 1991; Santolik and Gurnett, 2003) or from high latitudes.

In this paper, the chorus emissions recorded in large numbers during the night of 7-8 March 1986 are analysed. These chorus emissions were recorded during the strong magnetic storm period of 6-9 March 1986, with minimum $D_{s t}$-index of $-84 \mathrm{nT}$ on 6 March. The $D_{s t}$-index variations during the storm period are shown in Fig. 1, in which the observation period of chorus events is also marked. The most intense chorus emissions were registered during the night of 7-8 March, when the magnetic activity was highest $\left(\Sigma K_{p}=34\right)$ during the recovery phase of substorm.

Typical frequency-time spectrograms of the VLF chorus emissions recorded during nighttime, between 21:26 UT to 01:23 UT, on 7-8 March 1986 at Gulmarg, is shown in Fig. 2. The hiss is seen as an unstructured signal below about $2.5 \mathrm{kHz}$, and the discrete rising tones are chorus emissions. It is also observed that each chorus element, as shown in the figure, originates from the upper edge of the underlying hiss band. In general, the chorus occurrence rate and intensity is found to be closely correlated with the intense hiss activity. These chorus events started at 21:26 UT (02:56 LT) with an upper boundary frequency $f_{U B}$ of $5.8 \mathrm{kHz}$. Figure $2 \mathrm{a}$ contains five chorus risers observed at 21:26 UT in the frequency range $2.4-5.8 \mathrm{kHz}$. The average spacing between the traces is about $0.28 \mathrm{~s}$ but the third and fourth traces are only separated by $0.06 \mathrm{~s}$. It is not clear to us if these two traces may be caused by a single wave packet that is split up into two ducts that have slightly different propagation time, or if these traces represent different chorus elements. Over the course of time the upper boundary frequency began to increase and it reached $7.2 \mathrm{kHz}$ by $22: 20 \mathrm{UT}$, as shown in Fig. 2b. Here, a clear periodic structure with a period of about $0.29 \mathrm{~s}$ appears. For the next two hours the upper boundary frequency remained the same. From 00:20 UT, it began to slowly decrease and by 01:23 UT reached $4.6 \mathrm{kHz}$, as shown in Fig. 2c. The whole chorus event lasted for more than $4 \mathrm{~h}$. The observed mean chorus element has the following parameters: $f_{\text {min }}=2.4 \mathrm{kHz}, f_{U B}=6.5 \mathrm{kHz}$, frequency sweep rate, $\mathrm{d} f / \mathrm{d} t=40 \mathrm{kHz} / \mathrm{s}$ and successive repetition period, $T=0.3 \mathrm{~s}$. 


\section{Generation mechanism}

Considerations of the magnetosphere as a giant cyclotron maser (Trakhtengerts, 1979) are fruitful for describing the origin of different types of VLF emissions (Bespalov and Trakhtengerts, 1986). In the magnetosphere cyclotron maser (MCM) concept, separate magnetic flux tubes are considered as resonant cavities for whistler waves with conjugate ionospheres as mirrors for electromagnetic waves. Whistler wave packets propagating between mirrors amplify during interactions with trapped energetic electrons. In such a system there are different generation regimes (stationary regime with hiss generation, relaxation oscillation regime with generation of quasi-periodic emission, and a spike-like generation regime) which are similar to laboratory masers. However, mechanisms responsible for the origin of chorus temporal succession rate, the connection with hiss and the formation of the spectrum of separate elements cannot be explained by the simple MCM concept.

Recently, Trakhtengerts $(1995,1999)$ suggested a new generation regime of $\mathrm{MCM}$, the backward wave oscillator (BWO) generation regime. This regime was discovered in electronic devices many years ago (Johnson, 1955). The characteristic feature of such devices is that the waves propagate opposite to the motion of interacting electrons. Such a situation is realised in the cyclotron resonance:

$\omega-\omega_{B}=k v$,

where, $\omega_{B}$ is the electron gyrofrequency, $\omega$ is the wave frequency, $k$ and $v$ are the components of the wave vector and electron velocity along the magnetic field. MCM operates on the same resonance and serves as a cosmic analog to laboratory cyclotron resonance masers.

However, certain conditions have to be satisfied for a generator to operate in the BWO regime. The first condition requires that the phase velocity component along the magnetic field should be opposite to the electron motion. According to Eq. (1), this condition is satisfied if $\omega<\omega_{B}$.

The second condition is the existence of a well organised electron beam with small velocity dispersion in the region of discrete emission generation. This condition poses a significant problem, since there is no obvious reason for such a beam to be formed. The solution of this problem can be related to the fact that cyclotron interaction of energetic electrons with band-limited natural ELF/VLF noiselike emissions results in the formation of a specific steplike feature in the electron distribution function (Nunn and Sazhin, 1991). This step-like deformation of energetic electron distribution function ensures a large growth rate $\gamma_{H D}$ of whistler waves and transition to the BWO regime. Trakhtengerts (1995) showed that the step-like deformation of the distribution function in the magnetosphere can play the same role as a well organised beam in laboratory devices. The magnetospheric BWO has no fixed boundaries, and its interaction length $l$ is determined by the inhomogeneity of the geomagnetic field. According to Helliwell (1967) and Trakht- engerts $(1995,1999)$, the interaction length $l$ between the whistler waves and energetic electrons is limited by

$l_{0}<l<l_{m}$

with

$l_{0}=\left(\frac{L^{2} R_{E}^{2}}{k}\right)^{1 / 3}$

and

$l_{m}=L R_{E}\left(\sqrt{1+4\left(f_{U B}-f_{\min }\right) / f_{B}}-1\right)^{1 / 2}$.

Here, $R_{E}$ is the Earth's radius, $L$ is the McIlwain parameter. In terms of the electron plasma frequency $\omega_{p}=2 \pi \mathrm{f}_{p}$ and the gyrofrequency $\omega_{B}=2 \pi \mathrm{f}_{B}$, the wave number $k$ is given by the whistler dispersion relation as $k=\omega^{1 / 2} \omega_{p} c^{-1} /\left(\omega_{B}-\omega\right)^{1 / 2}$. Here, $c$ is the velocity of light.

The BWO generation starts when the beam density exceeds some threshold value. With a further increase in the beam density, the BWO regime changes from a continuous regime to a periodic regime, which is accompanied by a periodic modulation of wave intensity, and then to stochastic modulation. According to Trakhtengerts (1995), the BWO threshold condition can be written as

$p=2 \gamma_{H D} l /\left(\pi\left(v v_{g}\right)^{1 / 2}\right) \cong 2$,

where $\gamma_{H D}$ is the hydrodynamic growth rate in the case of a distribution function with a step-like deformation, and $v_{g}$ is the group velocity of the whistler waves. The growth rate of BWO generation with periodic modulation can be written as function of $p$ in the form (Trakhtengerts, 1995):

$\gamma_{B W O}=2 p(p-1) / T$,

where

$T=1.5 l\left(v_{g}^{-1}+v^{-1}\right)$

is the modulation period, which is connected with the repetition period of chorus elements.

After the transition to the BWO regime, the dynamical spectrum of a separate chorus element is formed similar to discrete signals triggered by VLF transmitters (Nunn, 1974). According to computer simulation of triggered emissions (Nunn, 1974; Omura et al., 1991) the optimal condition for the generation of chorus emissions, corresponding to the maximum value of the nonlinear growth rate, can be written as

$0.2 \leq|S| \leq 0.8$

where

$S=\Omega_{t r}^{-2}\left[3 v / 2\left(\partial \omega_{B} / \partial z\right)-\left(1+\omega_{B} / 2 \omega\right) \mathrm{d} \omega / \mathrm{d} t\right]$

and the trapping frequency $\Omega_{t r}$ is determined by the expression:

$\Omega_{t r}=\left(k u \omega_{B} b\right)^{1 / 2}$. 
Here, $b=B \sim / B_{L}$, where $B \sim$ is the whistler wave magnetic field amplitude, $B_{L}$ is the geomagnetic field and $u$ is the electron velocity component across the geomagnetic field. We can estimate the wave amplitude $B \sim$ from the relation,

$\Omega_{t r} / \gamma_{B W O} \cong 32 / 3 \pi$

which is valid in the case of an absolute instability (Trakhtengerts, 1984). From Eqs. (7) and (8) we can estimate the wave amplitude $B_{\sim}$ as

$B_{\sim}=(32 / 3 \pi)^{2} \gamma_{B W O}^{2} B_{L} /\left(k u \omega_{B}\right)$.

In our case the generated wave propagates from the equator towards the increasing magnetic field, so $S<0$ (Omura et al., 1991). Putting $S=-0.5$ and substituting into Eq. (7) the value of $\Omega_{t r}$, we get the frequency sweep rate as (Trakhtengerts, 1999)

$\mathrm{d} f / \mathrm{d} t=1.5 \omega \gamma_{B W O}^{2}\left(1+S_{0}\right) /\left(\omega_{B}+2 \omega\right)$,

where

$f=\omega / 2 \pi, S_{0}=v /\left(3 \gamma_{B W O}^{2}\right)\left(\partial \omega_{B} / \partial z\right)$.

To test this theory of the generation mechanism we have computed various chorus emission parameters using the above Eqs. (1)-(10) and compared them with our observations.

\section{Results and discussions}

The observations of VLF chorus emissions at low-latitude station Gulmarg show that, although chorus emissions are rare under quiet conditions, the occurrence rate of chorus emissions is high during magnetic storms. From the four hours of chorus observations during the storm of 7-8 March, we have selected three different intervals of chorus data for detailed study. The spectral analysis of the chorus events suggests that these emissions are likely to have originated from the hiss band (Hattori et al., 1991). Based on the direction finding measurements Hattori and Hayakawa (1994) have also suggested that a chorus event is triggered from a wavelet existing at the upper edge of the hiss band through a coherent wave-particle interaction. Observations of chorus events by satellites near the geomagnetic equator (Tsurutani and Smith, 1974) support the idea that the source of chorus emissions is localized most probably near equatorial region. By analysing POLAR satellite data Lauben at al. (2002) have studied various source characteristics of chorus and indicated the likely source region near the magnetic equator.

To explain the various features of chorus emissions recorded at Gulmarg, we compare our observations with the generation mechanism based on the BWO regime of the magnetospheric cyclotron maser (Trakhtengerts, 1995, 1999). During a magnetic storm, energetic electrons are injected into regions where the conditions for cyclotron instability are favorable. Such regions are the plasmapause and ducts with enhanced background plasma density. At the preliminary stage of the cyclotron instability, a sharp gradient in the energetic-electron velocity distribution function is formed. Recently, Pasmanik et al. (2002) have shown that step-like features in the velocity distributions arises naturally owing to interactions with noise-like VLF hiss, and these steps favour the amplification of whistler-mode waves propagating along the Earth's magnetic field lines. After the step formation, the stage with periodic generation of chorus elements starts. The BWO regime of generation may explain features of chorus emissions, such as the appearance of a succession of chorus elements, their relation to hiss, and their frequency-time spectrum.

Smirnova et al. (1976) have argued that the source of chorus emissions is extremely localised in space and the appearance of chorus at a wide range of latitudes is the result of a propagational effect. The outer boundary of the plasma pause has a focusing property for the VLF chorus emissions, and the maximum of chorus intensity is found to move toward lower latitudes with the increase in geomagnetic activity (Smirnova, 1984). The range of observed chorus frequencies is controlled by the equatorial electron gyrofrequencies (Burtis and Helliwell, 1976). We have used the upper boundary frequency $\left(f_{U B}\right)$ method developed by Smirnova (1984), to find out the location of source for the chorus emission events. The upper boundary frequency of the ground-based VLF chorus emissions is determined as the half equatorial electron gyrofrequency in the generation region, irrespective of the latitude of the observation station. Assuming a dipolar geomagnetic field, the $L$-value of the chorus emission source is then computed as (Smirnova, 1984)

$L=\left(440 / f_{U B}\right)^{1 / 2}$,

where $f_{U B}$ is in $\mathrm{kHz}$. Using the above method the $L$-value of the source region for the reported event of chorus emission is found to be $L_{\text {source }}=4.07$. The high $L$-value of the source compared to that of Gulmarg $(L=1.28)$ shows that the waves have propagated towards significantly lower latitudes, as expected during strong geomagnetic activity (Smirnova, 1976, 1984).

To examine the generation mechanism we have computed various chorus emission parameters using the above Eqs. (1)-(11). The magnetic field at the source is about $\mathrm{B}_{L}=4.6 \times 10^{-7} \mathrm{~T}$, and the equatorial electron gyrofrequency is $f_{B} \sim 13 \mathrm{kHz}$. From the Carpenter and Anderson (1992) empirical equatorial electron density profile evaluated for a plasmapause at $\mathrm{L}=4.0-4.1$ we find the electron density to be $\sim 16$ electrons $\mathrm{cm}^{-3}$ and the corresponding plasma frequency is $f_{p} \sim 36 \mathrm{kHz}$. Considering a frequency $f=f_{B} / 3$ we find from the dispersion relation that $k \sim 0.5 \mathrm{~km}^{-1}$. From Eq. (2) the interaction length $l$ should be between $l_{0}=1095 \mathrm{~km}$ and $l_{m}=17000 \mathrm{~km}$. Using the resonance condition (1) we find that the group velocity, $v_{g} \sim 2 \omega^{2} /\left(\omega_{B} k\right) \sim 3.6 \times 10^{7} \mathrm{~m} / \mathrm{s}$ is lower than the velocity of resonant electrons, $v \sim 1.1 \times 10^{8} \mathrm{~m} / \mathrm{s}$. The modulation period, which determines the repetition period of discrete elements, is then found from Eq. (5) to be between $0.06 \mathrm{~s}$ and $0.94 \mathrm{~s}$. Our experimental value of $\mathrm{T}=0.29 \mathrm{~s}$ is in this interval, and suggests that the real interaction length is around $5000 \mathrm{~km}$. 
Using the value of $T=0.29$ and $p=2$ in Eq. (4), we find the growth rate, $\gamma_{B W O}=16 \mathrm{~s}^{-1}$. This rather low growth rate, compared to, for example, $\gamma_{B W O}=50 \mathrm{~s}^{-1}$ for $L=4$ found by Demekhov and Trakhtengerts (2001), is consistent with an interaction length significantly larger than $l_{0}$. The low growth rate also implies that we should consider $S_{0}>>1$ when determining the frequency sweep rate from Eq. (10). Adopting a parabolic approximation for the magnetic field near the equatorial plane we have $\partial \omega_{\mathrm{B}} / \partial z=9 \omega_{B}\left(R_{E} L\right)^{-2} v t$, where $v=\left(\omega_{B} / \omega-1\right)^{3 / 2} \omega c / \omega_{p}$ is the velocity of the resonant electrons. The frequency sweep rate is then found to be

$$
\frac{d f}{d t}=4.5 \frac{\omega_{B}\left(\omega_{B}-\omega\right)^{2} c^{2}}{\omega_{p}\left(\omega_{B}+2 \omega\right) L^{2} R_{E}^{2}} t
$$

Comparing with observations we estimate from Fig. 2 that the frequency of the emissions increases from $2.4 \mathrm{kHz}$ to $4 \mathrm{kHz}$ in $0.055 \mathrm{~s}$ and reaches $6.5 \mathrm{kHz}$ after $0.1 \mathrm{~s}$. In the lower frequency range, centered on $f_{B} / 4$, we find that the sweep rate is about $29 \mathrm{kHz} / \mathrm{s}$. The formula above yields $d f / d t=t \times$ $1800 \mathrm{kHz} / \mathrm{s}^{2}$ at $f_{B} / 4$, and the theoretical sweep rate agrees with the observed average when $t=0.016 \mathrm{~s}$. The observed frequency sweep rate in the upper frequency band, centered around $5 f_{B} / 13$, is $55 \mathrm{kHz} / \mathrm{s}$. At this frequency Eq. (12) yields $d f / d t=t \times 844 \mathrm{kHz} / \mathrm{s}^{2}$, which agrees with the observed rate at $\mathrm{t}=0.065 \mathrm{~s}$. Notice that the frequency dependence of the coefficient in Eq. (12) counteracts the explicit parabolic time dependence, and allows the chorus elements to rise steeply from the background hiss.

Comparing the repetition period and frequency sweep rate in our low-latitude chorus observations with predictions from the BWO model, we find that they are generally consistent. The theoretical estimate for the repetition period is rather rough, but the observed period is well centered in the predicted interval. The average frequency sweep rate calculated from theory is consistent with the observations, and the theory also predicts the observed increase of the sweep rate with increasing frequency. Thus, we have confirmed that the BWO model is consistent with a detailed analysis of chorus spectra recorded at low latitudes.

\section{Conclusion}

A generation mechanism for various temporal and spectral features of VLF chorus emissions recorded at the lowlatitude ground station Gulmarg is presented on the basis of the backward wave oscillator regime of the magnetospheric cyclotron maser. On the one hand, this model connects directly with the theory of triggered VLF emissions, and on the other hand, it explains peculiarities of chorus emissions, such as its connection with hiss, the appearance of a succession of chorus elements with small repetition frequency and amplitude of a signal. On the basis of this theory, various chorus emission parameters, as well as some magnetospheric parameters relevant to the generation process, are computed. These parameters are comparable to that of the observed values which support the possibility for wide use of VLF chorus data for the ground-based diagnostics of the state of the magnetospheric plasma during substorm.

Further experimental and theoretical studies of VLF chorus emissions are required for a complete understanding of this phenomenon. More analytical and computational calculations are also needed with more strict considerations of the magnetic field inhomogeneity.

Acknowledgements. A. K. Singh thanks Department of Science and Technology, New Delhi, India for providing BOYSCAST Fellowship and Department of Theoretical Space Physics, Umeå University, Sweden for providing excellent facility to work.

Topical editor T. Pulkkinen thanks M. Parrot for his help in evaluating this paper.

\section{References}

Bespalov, P. A. and Trakhtengerts, V. Y.: The cyclotron instability of the Earth's radiation belts, Reviews of Plasma Physics, edited by Leontovich, M. A., vol 10, 155-292, Plenum, New York, 1986.

Burtis, W. J. and Helliwell, R. A.: Magnetospheric chorus: Occurrence patterns and normalized frequency, Planet. Space Sci., 24, 1007-1024, 1976.

Carpenter, D. L. and Anderson, R. R.: An ISEE/whistler model of equatorial density in the magnetosphere, J. Geophy. Res., 97, 1097-1108, 1992.

Cornilleau-Wehrlin, N., Gendrin, R., Lefeuvre, F., Parrot, M., Grard, R., and Jones, D., VLF waves observed onboard GEOS-1, Space Sci. Rev., 22, 371-382, 1978.

Demekhov, A. G. and Trakhtengerts, V. Y.: Theory of generation of discrete ELF/VLF emissions in the Earth's magnetosphere, Radiophys. Quant. Elec., 44, 1-2, 103-116, 2001.

Hattori, K., Hayakawa, M., Lagoutte, D., Parrot, M., and Lefeuvre, F.: Further evidence of triggered chorus emissions from wavelets in the hiss band, Planet. Space Sci., 39, 1465-1472, 1991.

Hattori, K. and Hayakawa, M.: Consideration of dynamic spectra and direction finding results of hiss-triggered chorus emissions, Proc. NIPR Symp. Upper Atmos. Phys., 7, 40-52, 1994.

Helliwell, R. A.: Whistlers and Related Ionospheric Phenomena, Stanford Univ. Press, Stanford, USA, 1965.

Helliwell, R. A.: A theory of discrete emissions from the magnetosphere, J. Geophys. Res., 72, 4773-4790, 1967.

Johnson, H. R.: Backward wave oscillator, Proc. IEEE, 43, 684, 1955.

Lalmani, Babu, M. K., Kumar, R., Singh, R., and Gwal, A. K.: An explanation of daytime discrete VLF emissions observed at Jammu $(L=1.17)$ and determination of magnetospheric parameters, Ind. J. Phys., 74B, 2, 117-123, 2000.

Lauben, D. S., Inan, U. S., Bell, T. F., and Gurnett, D. A.: Source characteristics of ELF/VLF chorus, J. Geophys. Res., 107, A12, 1429, doi:10.1029/2000JA003019, 2002.

Nunn, D.: A self-consistent theory of triggered VLF emissions, Planet. Space Sci., 22, 349-378, 1974.

Nunn, D.: A nonlinear theory of sideband stability in ducted whistler modes, Planet. Space Sci., 34, 5, 429-451, 1986.

Nunn, D. and Sazhin, S. S.: On the generation mechanism of hisstriggered chorus, Ann. Geophys., 9, 603-613, 1991.

Omura, Y., Nunn, D., Matsumoto, H., and Rycroft, M. J.: A review of observational, theoretical and numerical studies of VLF triggered emissions, J. Atmos. Terr. Phys., 53, 351-368, 1991. 
Pasmanik, D. L., Demekhov, A. G., Nunn, D., Trakhtengerts, V. Y., and Rycroft, M. J.: Cyclotron amplification of whistlermode waves: A parametric study relevant to discrete VLF emissions in the Earth's magnetosphere, J. Geophys. Res., 107, A8, doi:10.1029/2001JA000256, 2002.

Santolik, O. and Gurnett, D. A.: Transverse dimensions of chorus in the source region, Geophy. Res. Lett., 30, 2, 1031, doi:1029/2002GL016178, 2003.

Sazhin, S. S.: Natural Radio Emissions in the Earth's Magnetosphere, Nauka, Moscow, 1982.

Sazhin, S. S. and Hayakawa, M.: Magnetospheric chorus emissions: a review, Planet. Space Sci., 40 , 681-697, 1992.

Singh, R. P.: Whistler studies at low latitudes: a review, Ind. J. Rad. Spa. Phys., 22, 139-155, 1993.

Singh, A. K., Singh, D. K., Patel, R. P., Singh, R. P., Singh, A. K.: Two types of ELF hiss observed at Varanasi, India, Ann. Geophys., 17, 1260-1267, 1999.

Singh, R., Patel, R. P., Singh, R. P., and Lalmani: An experimental study of hiss-triggered chorus emissions at low latitude, Earth Planets Space, 52, 37-40, 2000.

Smirnova, N. A., Novikov, Yu. P., Kleimenova, N. G., and Titova, E. E.: Some spectral peculiarities of VLF emissions registered on the Earth surface near the plasmapause projection, J. Atmos. Terr. Phys., 38, 1217, 1976.
Smirnova, N. A.: A fine structure of the ground observed VLF chorus as an indicator of the wave particle interaction process in the magnetosphere, Planet. Space Sci., 32, 4, 425-438, 1984.

Smith, A. J., Freeman, M. P., and Reeves, G. D.: Post midnight VLF chorus events, a substorm signature observed at the ground near L = 4, J. Geophy. Res., 101, A11, 24 641-24 653, 1996.

Trakhtengerts, V. Y.: Magnetosphere as an Alfven maser, Earth Universe, 4, 42, 1979.

Trakhtengerts, V. Y.: Relaxation of plasmas with anisotropic velocity distribution, Handbook of Plasma Physics, vol 2, Basic Physics II, edited by Galeev, A. A. and Sudan, R. N., 519-552, Elsevier, New York, 1984.

Trakhtengerts, V. Y.: Magnetosphere cyclotron maser: Backward wave oscillator generation region, J. Geophys. Res., 100, A9, 17 205-17 210, 1995.

Trakhtengerts, V. Y., Rycroft, M. J., and Demekhov, A. G.: Interrelation of noise-like and discrete ELF/VLF emissions generated by cyclotron interactions, J. Geophys. Res., 101, A6, $13293-$ 13 303, 1996.

Trakhtengerts, V. Y.: A generation mechanism for chorus emission, Ann. Geophys., 17, 95-100, 1999.

Tsurutani, B. T. and Smith, E. J.: Post midnight chorus: A substorm phenomenon, J. Geophys. Res., 79, 118-127, 1974. 